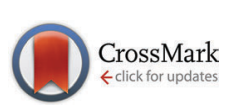

Cite this: Phys. Chem. Chem. Phys., $2016,18,23412$

DOI: $10.1039 / c 6 c p 90200 f$

www.rsc.org/pccp

\section{Correction: Tuning the Schottky contacts in the phosphorene and graphene heterostructure by applying strain}

\author{
Biao Liu, ${ }^{a}$ Li-Juan Wu, ${ }^{a}$ Yu-Qing Zhao, ${ }^{a}$ Lin-Zhi Wang ${ }^{a}$ and Meng-Qiu Cai ${ }^{\text {ab }}$
}

Correction for 'Tuning the Schottky contacts in the phosphorene and graphene heterostructure by applying strain' by Biao Liu et al., Phys. Chem. Chem. Phys., 2016, 18, 19918-19925.

The name of the corresponding author contains a spelling mistake. The corresponding author should be written as "Meng-Qiu Cai" and not as "Meng-Qiu Caii".

This error does not affect the discussion and conclusion.

The Royal Society of Chemistry apologises for these errors and any consequent inconvenience to authors and readers.

\footnotetext{
${ }^{a}$ School of Physics and Electronics Science, Hunan University, Changsha, Hunan 410082, China. E-mail: mqcai@hnu.edu.cn; Tel: +8673188821177

${ }^{b}$ Synergetic Innovation Center for Quantum Effects and Applications (SICQEA), Hunan Normal University, Changsha 410081, China
} 